\title{
A Case of Suspected Breast Cancer Metastasis to Brachial Plexus Detected by Magnetic Resonance Neurography
}

\author{
Atsushi Mizuma Chikage Kijima Eiichiro Nagata Shunya Takizawa \\ Department of Neurology, Tokai University School of Medicine, Isehara, Japan
}

\section{Keywords}

Magnetic resonance neurography $\cdot$ Brachial plexus $\cdot$ Breast cancer $\cdot$ Metastasis

\begin{abstract}
Metastasis of breast cancer is often detected through a long-term course and difficult to diagnose. We report a case of brachial plexopathy suspected to be the initial lesion of breast cancer metastasis, which was only detected by magnetic resonance (MR) neurography. A 61year-old woman was admitted to our hospital within 2 years after operation for breast cancer because of progressive dysesthesia and motor weakness initially in the upper limb on the affected side and subsequently on the contralateral side. Enhanced computed tomography, axillary lymph node echo, gallium scintigraphy, and short tau inversion recovery MR images showed no abnormalities. MR neurography revealed a swollen region in the left brachial plexus. We suspected neuralgic amyotrophy and initiated treatment with intravenous immunoglobulin therapy and steroid therapy. However, there was no improvement, and the progression of motor weakness in the bilateral lower limbs appeared over 4 years. Concomitant elevation of carbohydrate antigen $15-3$ level $(58.9 \mathrm{U} / \mathrm{ml})$ led us to suspect breast cancer metastasis, which was associated with the worsening of neurological findings, although gallium scintigraphy and bone scintigraphy showed no inflammatory and metastatic lesions. Swelling of the cauda equina in enhanced lumbar MR imaging and abnormal accumulation at the brachial plexus and cervical spinal cord in positron-emission tomography were newly detected contrary to the normal findings on the gallium scintigraphy, which suggested cerebrospi-
\end{abstract}


nal fluid seeding. We suspected breast cancer metastasis about the initial brachial plexopathy based on the clinical course. MR neurography may be a helpful tool to detect metastatic lesion, especially in nerve roots.

\section{Introduction}

Brachial plexopathy-associated breast cancer can occur by radiation injury or metastatic spread of the tumor [1]. This plexopathy can be detected by short tau inversion recovery (STIR) magnetic resonance (MR) imaging (MRI) and fluorodeoxyglucose positron-emission tomography (PET) $[2,3]$. On the other hand, metastasis of breast cancer is often detected through a long-term course and difficult to diagnose [4]. Here, we report a case of brachial plexopathy suspected to be the initial lesion of breast cancer metastasis, which was only detected by MR neurography.

\section{Case and Discussion}

A 61-year-old woman was admitted to our hospital within 2 years after operation for breast cancer (invasive ductal carcinoma, T2N0M0, stage IIA) because of progressive dysesthesia and motor weakness initially in the upper limb on the affected side and subsequently on the contralateral side. Laboratory findings showed no elevation of autoantibodies (including anti-ganglioside antibodies and anti-neural antibodies) or tumor markers [carcinoembryonic antigen $1.7 \mathrm{ng} / \mathrm{ml}$, carbohydrate antigen (CA) 15-3 $22.0 \mathrm{U} / \mathrm{ml}$. Cerebrospinal fluid (CSF) also showed no elevation of protein and pleocytosis and class I of cytology. Electromyographic study showed the extension with the motor conduction velocity on the left side of median $(42.8 \mathrm{~m} / \mathrm{s})$ and ulnar $(47.5 \mathrm{~m} / \mathrm{s})$ nerves. Compound muscle action potentials were normal and conduction block was not shown. Sensory nerve action potentials were absent. Needle electromyography showed neurogenic changes along with the voluntary movement on the upper extremities and denervation potential at rest. Enhanced computed tomography, axillary lymph node echo, gallium scintigraphy, and STIR MRI images showed no abnormalities (fig. 1). MR neurography, which can detect nerve swelling and edema [5], revealed a swollen region in the left brachial plexus (fig. 2). At first, we suspected neuralgic amyotrophy and initiated treatment with intravenous immunoglobulin therapy and steroid therapy. However, there was no improvement, and motor weakness gradually progressed and also appeared in the lower limbs over 4 years. Concomitant elevation of CA15-3 level $(58.9 \mathrm{U} / \mathrm{ml})$ led us to suspect breast cancer metastasis, which was associated with the worsening of neurological findings, although gallium scintigraphy and bone scintigraphy showed no inflammatory and metastatic lesions. On the other hand, CSF showed elevated protein $(465 \mathrm{mg} / \mathrm{dl})$ and pleocytosis $\left(11 / \mathrm{mm}^{3}\right)$ in parallel with the increased CA15-3 levels. Cytology also showed class III with atypical cells. Swelling of the cauda equina in enhanced lumbar MRI (fig. 3) and abnormal accumulation at the brachial plexus and cervical spinal cord in PET (fig. 4) were newly detected contrary to the normal findings on the gallium scintigraphy, which suggested CSF seeding. As reported previously about the high false-negative rate of malignant cells by CSF cytology [6], we could not detected metastatic malignant cells in CSF. But the abnormal lesions in enhanced MRI [7] and PET suggested malignant tumor. We suspected as the initial brachial plexopathy due to breast cancer metastasis based on the clinical course; CSF seeding and the elevation of CA15-3 in parallel with the worsening of brachial 
Mizuma et al.: A Case of Suspected Breast Cancer Metastasis to Brachial Plexus Detected by Magnetic Resonance Neurography

plexopathy. Although STIR imaging did not show abnormal findings in our case, MR neurography may be a helpful tool to detect metastatic lesion, especially in nerve roots [8].

\section{Disclosure Statement}

The authors declare no conflict of interest.

\section{References}

1 Zingale A, Ponzo G, Ciavola G, et al: Metastatic breast cancer delayed brachial plexopathy. A brief case report. J Neurosurg Sci 2002;46:147-149.

-2 Hoeller U, Bonacker M, Bajrovic A, et al: Radiation-induced plexopathy and fibrosis. Is magnetic resonance imaging the adequate diagnostic tool? Strahlenther Onkol 2004;180:650-654.

-3 Ho L, Henderson R, Luong T, et al: 18F-FDP PET/CT appearance of metastatic brachial plexopathy involveing epidural space from breast carcinoma. Clin Nucl Med 2012;37:e263-e264.

4 Joensuu H, Pylkkanen L, Toikkanen S: Late mortality from pT1N0M0 breast carcinoma. Cancer 1999;85:2183-2189.

5 Thawait SK, Chaudhry V, Thawait GK, et al: High-resolution MR neurography of diffuse peripheral nerve lesions. Am J Neuroradiol 2011;32:1365-1372.

-6 Glants MJ, Cole BF, Glants LK, et al: Cerebrospinal fluid cytology in patients with cancer: minimizing false-negative results. Cancer 1998;82:733-739.

7 Chamberlain M, Sandy A, Press G: Leptomeningeal metastasis. A comparison of gadolinium-enhanced MR and contrast-enhanced CT of the brain. Neurology 1990;40:435-438.

-8 Yuh EL, Jain Palrecha S, Lagemann GM, et al: Diffusivity measurements differentiate benign from malignant lesions in patients with peripheral neuropathy or plexopathy. Am J Neuroradiol 2015;36:202-209. 


\section{Case Reports in Oncology}

\begin{tabular}{l|l}
\hline Case Rep Oncol 2016;9:395-399 \\
\hline 10.1159/000447054 & $\begin{array}{l}\text { @ 2016 The Author(s). Published by S. Karger AG, Basel } \\
\text { www.karger.com/cro }\end{array}$ \\
\hline
\end{tabular}

Mizuma et al.: A Case of Suspected Breast Cancer Metastasis to Brachial Plexus Detected by Magnetic Resonance Neurography

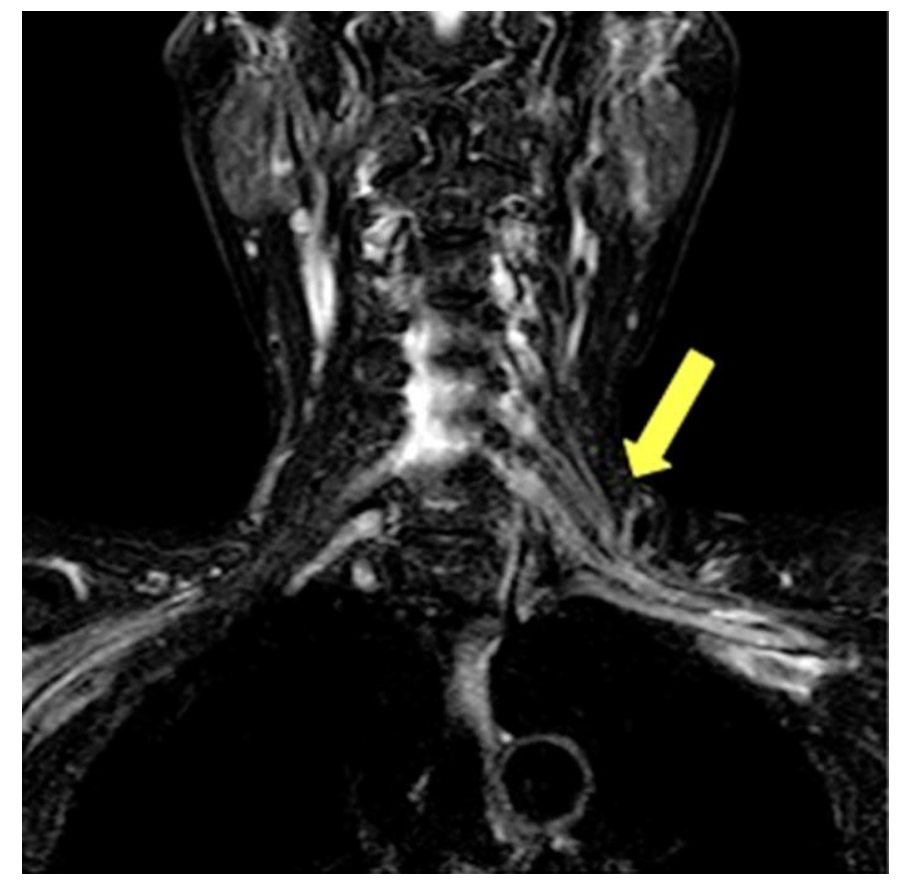

Fig. 1. STIR MRI showed no abnormalities.

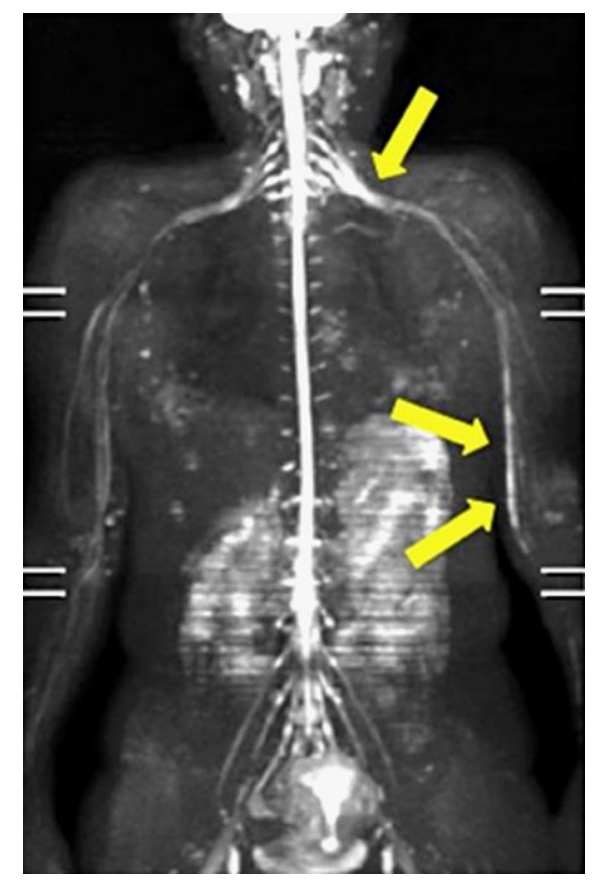

Fig. 2. MR neurography revealed a swollen region in the left brachial plexus. 


\section{Case Reports in Oncology}

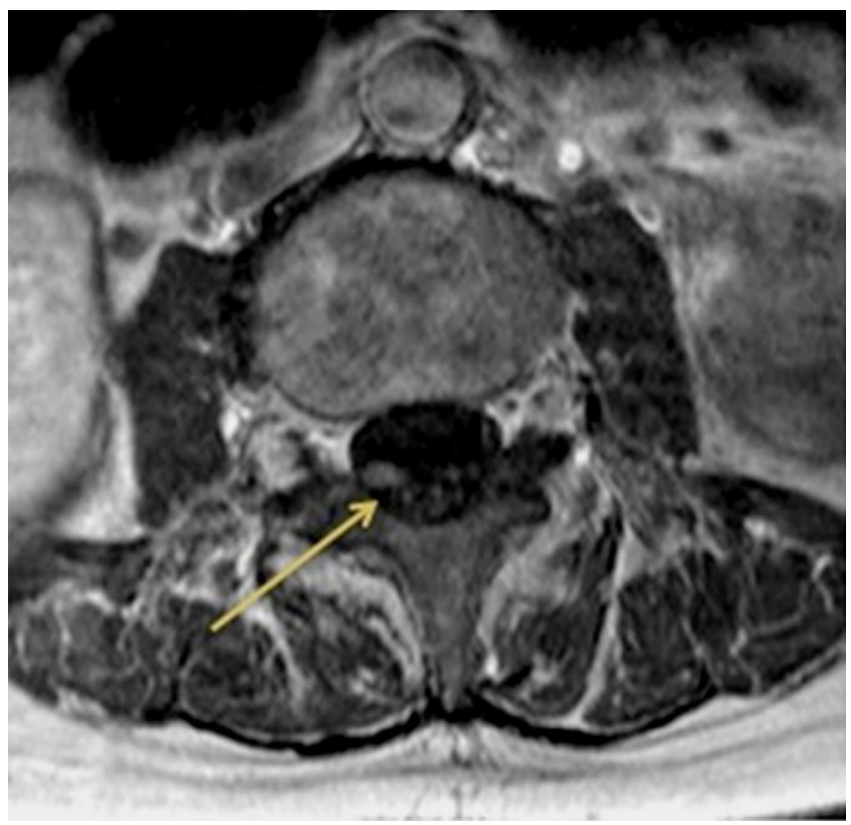

Fig. 3. Enhanced lumbar MRI showed the swelling of the cauda equina.

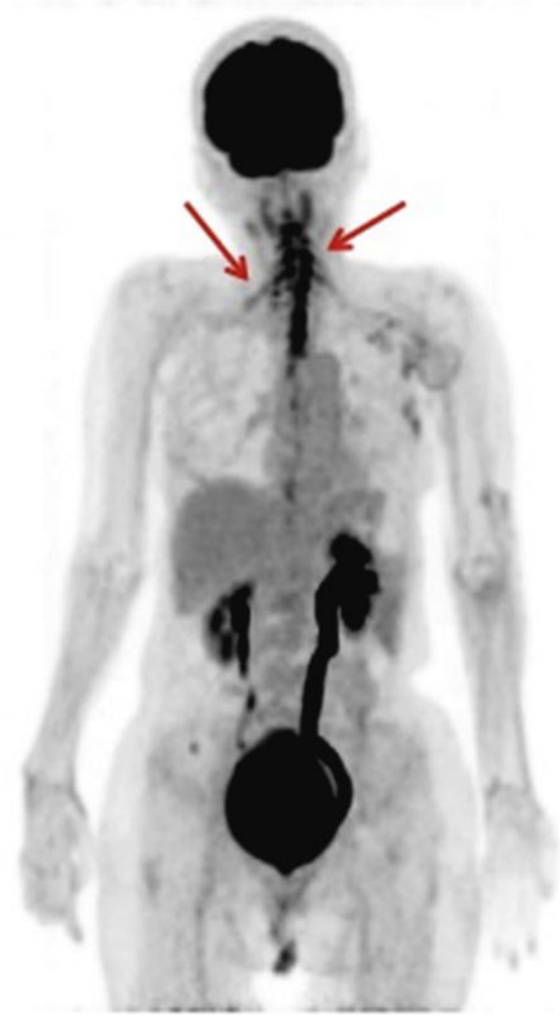

Fig. 4. Fluorodeoxyglucose PET showed abnormal accumulation at both the brachial plexus and cervical spinal cord. 